\title{
Simulation models of economic development: theoretical and methodological aspects
}

\author{
Olga Patrakeeva ${ }^{1, *}$ \\ ${ }^{1}$ Federal Research Center the Southern Scientific Centre of the Russian Academy of Sciences, \\ 344006, 41 Chehova str., Rostov-on-Don, Russia
}

\begin{abstract}
Regional socioeconomic development largely depends not only on objective factors (geographic position, natural resources, human potential) but also on subjective factors, in particular on the efficiency of actions taken by regional governing bodies to solve major problems of supporting economic growth and, therefore, personal income growth. The revision strategy for regional development is periodicity required on the strength of possible changes in the regional economy and the internal and external market situation. At present, simulation modelling is widely used in an effort to study regional processes. A simulation model built on the principles of systems dynamics is presented in this paper. In the next stage, a decision support system for the management of regional sustainable development was engineered.
\end{abstract}

\section{Introduction}

In many respects, regional socio-economic development depends on objective factors (geographical location, natural resources), although it depends on subjective factors to a greater extent (for example, on the efficiency of regional authorities). Regional authorities focus on the settlement of economic problems as a consequence of the problem of public welfare. Problemsolving depends on systematic and sustainable bases, especially in the unstable economic situation in the world (specifically, in the Russian Federation).

Even an appropriate regional strategy has a need for periodic adjustment by virtue of global economic changes and changes in internal and external markets and socio-economic conditions. There are many authority management tools that are aimed at the regulation of regional processes, such as socio-economic, ecological, scientific and technical forecasts; normative-legal acts, directed to economic development and improvement of the investment climate; public-administrative, ecological and market regulators; and federal and regional development programs of Russian administrative units.

There is no method for the elaboration and application of the above-listed tools without the adoption of modern decision support systems. In recent years, simulation models of socioeconomic systems have formed the basis of regional DSS. Simulation modelling provides a scientific basis for decision support systems.

\footnotetext{
* Corresponding author: olgapatrakeyeva@yandex.ru
} 
DSS on the basis of a computer simulation model enables users to work out various scenarios of regional development and to evaluate the consequences of their implementation in real time. Similarly, a DSS provides an opportunity to consider stochastic processes and check the consequences of decisions under the conditions of risk and uncertainty.

\section{Background}

\subsection{System dynamics in regional research}

Systems dynamics is one of the methods of simulation modelling. Systems dynamics was used as a modelling tool because of its rigorous approach in capturing interrelationships among variables and its handling of dynamic aspects of the system's behaviour. The application of this approach was made possible because of the success in designing management systems with feedback, progress in methods of computer modelling, the rapid and powerful growth of computer engineering, and the accumulated experience in modelling decision processes [33]. Systems dynamics allows us to create flight simulators that permit us to retrace, in real time, the consequences of decisions, comprehending the structure of the complex systems and designing tactics and strategies for more success [35, 36, 39].

Systems dynamic models are applied jointly with differential equations that are balanced as well as in combination with the principles and methods of logistics, which are founded on optimisation, control, and integrated flows in complex systems. Thus, under the leadership of J. Forrester, a systems dynamics model simulating the development of the American economy was generated. There was not an exogenous time series at the input. The behaviour of the economy was completely determined by the interaction of endogenous factors. Cycles that have a period of 3-7 years (the Kuznets cycles) might be observed in economic behaviour. In addition, it was possible to identify the nonlinear interaction effect of waves of different periods $[9,10]$.

In the 70's of the last century, a city dynamics model was developed by J. Forrester. This model was focussed on an analysis of urban territory evolution. In the framework of this dynamic model, three principal subsystems were determined: population (managers, partially employed and employed), housing stock and business. A growth model represented the city's behaviour as a system from the moment of occurrence on an empty land plot to complex reclamation and stable development. In the monograph [11, p. 14], various urban assistance programs were analysed. The model of the urban system made it clear that the latest and current programs had worsened the city.

One of the most famous systems dynamic models is the model WORLD-3, which was engineered under the direction of D. Meadows [25]. This model allows the study of longterm trends or systems such as interstate associations or even the world as a whole. Usually in global systems it is necessary to account for various crossing relationships of economic, political, social, demographic and other development factors.

The systems dynamic approach application in DSS allows joining several function spaces of studied objects into an integrated system and providing an organisational and quantitative basis for making effective management decisions.

\subsection{Scenario analysis in regional research}

It is important to understand in what way a region as a system will react in response to socioeconomic, political, and environmental changes. This type of understanding is necessary to create conditions that are conductive to the long-term economic growth of a region. This issue 
is a complex and compound problem that requires an interdisciplinary approach (for example, $[28,37])$.

These pioneering applications combines numerical modelling and scenario analysis. Each developed methodological approach proved its suitability for the evaluation of regional development. Furthermore, the approaches have been successfully used in different combinations (for example, $[1,3,38]$ ). Integrated numerical modelling involves the analysis of complex relationships through simulations that include socio-economic, ecological and regulatory issues. These models combine interdisciplinary approaches, and they are considered to be powerful tools for providing information in policy making and planning decisions in the cases of cognitive and institutional constraints on a decision unit maker (DUM) [13].

Various methods are applied to obtain quantitative estimates viz. extrapolation and the remaining forecasting methods based on the assumption of process stability. However, such methods do not allow considering problems that are specific to regional development because of uncertainty. Uncertainty includes the results of the variety of interactions between agents (consumer behaviour, regional marketing strategies and other aspects) as well as different levels of the decision-making hierarchy (international, regional, household and other levels).

Scenario analysis is one of the most commonly used and important techniques that allows accounting for uncertainty factors. Scenarios that are based on the current situation, logical and reasoned inferences about future events and their interactions describe potential options for further development of the system. There are probabilistic models and methods that are used for design and scenario analysis, such as stochastic differential equations, Monte Carlo methods, and Bayesian probabilistic networks [5, 23].

The specific character of scenario modelling is the simultaneous consideration of multiple development options with inherent opportunities and risks, subjective and objective, internal and external factors, criteria and indicators [7]. According to Herman Kahn, one of the founders of scenario method strategic thinking, we should get away from simple extrapolations based on thinking by analogy, which is undoubtedly important but does not give the level of detachment from reality that is necessary for strategic thinking [30].

The development of the analysis and design of a system is realised within the framework of term variants that represent a hypothetical sequence of events in an effort to focus attention on causal processes and decisions [14].

The main task of the scenario method is the substantiation of strategic management decisions. Flexible step-by-step correction decisions, action plans and conscious controlling have fundamental importance in this method [7]. This approach is used for anyone in the management sphere: from investment and staff management in small business to multinational corporations, governments, regions, countries and the world as a whole.

\subsection{Decision-making support systems}

DSS uses knowledge and theory from diverse areas such as database research, artificial intelligence, decision theory, economics, cognitive science, management science, mathematical modelling, and other fields [21].

Decision-Making Support Systems can be classified into a number of categories (Table 1).

This classification could be supplemented by Larichev's classification [22] and Holsapple and Whinston's classification [15].

The author's DSS presented in this paper is interactive. It allows interfering in the course of experiments and not passively waiting for model execution. The DSS is a modeldriven, information and research system for one user. It permits us to «count» short-term 
and long-term scenarios and forecasts of regional development. The system may be used for practical purposes.

The combination of simulation modelling and scenario approaches allows elaborate DSSs that are designed for solving problems such as forecasting and studying the possible consequences and efficiency of management decisions, which are alternatives in the building and selection of the optimal solution [20].

Table 1. Classification of DMSS.

\begin{tabular}{|c|c|}
\hline Classification criterion & Types \\
\hline $\begin{array}{c}\text { Mode of assistance } \\
{[31]}\end{array}$ & $\begin{array}{c}\text { Model-driven, Knowledge-driven, Document-Driven, Data- } \\
\text { Driven, Communications-Driven }\end{array}$ \\
\hline $\begin{array}{l}\text { Degree of action implication } \\
\text { of system output }[2]\end{array}$ & $\begin{array}{l}\text { File drawer systems, Data analysis systems, Analysis } \\
\text { information systems, Accounting and financial model-based } \\
\text { DSS, Representational model-based DSS, Optimisation model- } \\
\text { based DSS, Suggestion DSS based on logic models }\end{array}$ \\
\hline $\begin{array}{l}\text { Degree of intelligence data } \\
\text { processing }[6]\end{array}$ & $\begin{array}{c}\text { Information and research, operative and analytical DSS, } \\
\text { intelligent DSS }\end{array}$ \\
\hline $\begin{array}{c}\text { User interaction } \\
{[15][21]}\end{array}$ & Passive, active, cooperative DSS \\
\hline Users & DSS for many users; DSS for one user \\
\hline Managerial situation [4] & DSS for planning decisions; DSS for innovative decisions \\
\hline Reviewed period & $\begin{array}{l}\text { DSS review of available information on the object; DSS } \\
\text { predicting the future state of the object }\end{array}$ \\
\hline Purposing & DSS for theoretical purposes; DSS for practical purposes \\
\hline Planning period & Long-term, medium-term, short-term DSS \\
\hline Routing [27] & $\begin{array}{l}\text { DSS managing short-term behaviour of organisations, DSS } \\
\text { defining dynamics of changes in the company's capital; DSS } \\
\text { defining changes of functional characteristics in time }\end{array}$ \\
\hline Activity & Banking, insurance, trade (and other) DSS \\
\hline
\end{tabular}

Here are examples of already built DSSs that solve noted problems.

A two-layer DSS for rural sustainable development is presented in [16]. This system was developed for the comprehensive planning of socioeconomic development and ecoenvironment protection in Yongxin County, Jiangxi Province, China. This system integrates a dynamic database system, large-scale linear programming for regional managers and integer linear programming for farmers, which takes a step toward linking farmers' microplanning with administrators' macropolicies. The first level focuses on the social, economic and environmental variables. The goal of this level is to develop planning strategies that achieve maximum socioeconomic benefits and eco-environment quality on a macroscale. The second level is a functional portfolio analysis system for farmers. This system is designed to optimise individual economic behaviours.

The paper in [40] designs an agent-based regional agricultural economy decision support system (RAEDSS) to address complex decision problems. Dynamic analysis, evaluation, forecast, optimisation and decisions of regional agricultural economy are the central task of RAEDSS. Agent-based modelling is introduced to simulate and evaluate policy impact on rural development in different scenarios. The agent-based model shows that it is possible to simulate rural and agricultural behaviours of heterogeneous individual decision-makers to study overall characteristics of agricultural development. Moreover, there is an opportunity to research the 
macro evolution of an entire region and to provide a solid problem and foundation for future research on the interaction process of agricultural development and urbanisation.

The Core decision support system satisfies a requirement of the Sinaloa State Government in Mexico. Core performs analysing the competitiveness of Mexican regional entities. The competitiveness evaluation is considered to be a multicriteria sorting problem. In Core, the new outranking-based THESEUS method is applied to making the assignments of regional entities to competitiveness categories. This DSS is a tool that is placed at the disposal of the Government of Sinaloa and the remainder of the country. The core reflects in a robust way the level of competitiveness of different regions, allowing governments to better define their policies by locating the financial resources more efficiently [8].

Lychkina's information and analytical DSS is realised by means of Data Mining, regression, cluster analysis, intelligent technology, genetic algorithms, neural network technologies, and other tools. According to Lychkina's opinion, the important stage of the decision-making procedure is identifying key issues of the object domain and data analysis (Data Warehouse). Computed dependency visualising is realised by OLAP.

The model of complex regional socio-economic development consists of two levels:

1. Analysis and forecasting of regional resource potential. The first level includes blocks: natural resources, industrial funds, demography, finance, and external economic relations.

2. Management decisions elaboration. Strategy making is realised in the lines of investigation: industry of regional subordination, regional industrial and social infrastructure.

The simulation model, intelligence and expert systems are the basis of DSS. The intelligence and expert systems accumulate the experience of solving management problems and provide expert team participation. Experts have access to the model complex by a dialog interface [24].

An integrated modelling system for the simulation of the consequences of industrial accidents was developed by regional Civil Protection Department [34]. One of the system's modules, the decision support system, implements the algorithms for the management of offsite consequence analysis that allow a fast evaluation of the hazards that are associated with a given industrial plant. This DSS is a user-friendly system for environmental emergency situations, which is a useful tool for environmental decision makers. The main objective of the system is evaluating the impacts and potential management options for industrial accidents based on usersupplied information and decisions. If the necessary information is available, then the DSS will also be applied in other regions.You are free to use colour illustrations for the online version of the proceedings but any print version will be printed in black and white unless special arrangements have been made with the conference organiser. Please check whether or not this is the case. If the print version will be black and white only, you should check your figure captions carefully and remove any reference to colour in the illustration and text. In addition, some colour figures will degrade or suffer loss of information when converted to black and white, and this should be taken into account when preparing them.

\section{DSS oriented on regional development}

\subsection{The model}

The simulation model based on systems dynamics principles is elaborated here. The iThink software package, which is specifically produced for systems dynamics modelling [41], was used for working up the model and user-friendly interface of the DSS [17]. 
This simulation model allows conducting a multidimensional scenario stress testing economy to find the impact of the crisis factors. Computer simulation of the system behaviour permits the user to monitor selected characteristics of the system. With all of these factors occurring in the decision unit maker (DUM), changing the guiding principal within the system allows the opportunity to observe the likely behaviour modifications of the real system (the region).

The main problems, which are solved in the DSS framework, are:

1. Systematisation of available statistical information and extraction of targets that reflect the socio-economic situation of the Rostov region.

2. Modelling and analysis of the regional socio-economic situation.

3. Obtaining multivariate scenarios of the Rostov region's socio-economic development. There is an opportunity to realise retrospective analysis. In other words, DSS permits us to examine scenarios that could be accepted but were not realised.

The concept of model building assumes implementing the following main stages:

1. Analysis of a regional statistical database with a determination of the basic model parameters.

2. Definition and formalisation of parameter dependencies (the balance ratio, functional relationships) in accordance with the logic of economic indicators' relationships based on econometric modelling techniques.

3. Exogenous and endogenous dedication of model parameters.

4. Obtaining the information and a logic model, construction of its network structure based on identified relationships, exogenous and endogenous variables.

5. Building a decision support system based on an established model and its validation.

The proposed approach for model construction allows a combination of the complex system balance and functional relationships, a set of results indicators, and scenario modelling algorithms that enable the modelling and systemic presentation of the results. Simultaneously, the developed tool is adapted for regional management.

The model regional economic system is rated as a result of three institutional agents' interactions: the Producer, Consumer, and Regional Management Center. Small, medium and large-scale businesses are isolated in Producer.

Thus, the model is composed of the following building blocks or sectors (Fig. 2):

1. Small-Scale Business.

2. Medium and Large-Scale Businesses.

3. Population.

4. Prices and Taxes.

5. Consolidated Regional Budget.

6. Social and Economic Indicators.

The model includes a set of factors (further described in this paper) that relate to issues of regional socio-economic development.

Functional dependences of the parameters were revealed on the basis of econometric modelling, and balance ratios were also observed. The selection of the resulting indicators was conducted in accordance with the model structure and proceeded from the requirements of consistent output information and the availability of necessary statistics.

We singled out the following resulting indicators: the gross regional product, the real income of the population, the aggregate demand of the population, labour productivity, budget revenues, expenditures and balance. All of the identified relationships are displayed in the network graph. Furthermore, the network structure reflects information flows within the model. Retrospective information can be entered through dialog boxes. Decision unit makers can influence the calculation process by means of an operational definition of the scenario parameters and by controlling the impacts. 
iThink allows us to work with the simulation model at three levels: conceptual floworiented models, system dynamics equations and the specification of model parameters.

Total revenue, cost price and profit, employment, parameters of investment and tax policy, and sources of capital investment (owned and borrowed funds, government support) are presented in Small-Scale Business and Medium and Large-Scale Business blocks.

The main processes that account for feedback are modelled in these blocks [19, 32]:

1. Investment increase (as a consequence of equipment enhancement) permits us to enlarge the output and quality of the products. Accordingly, the total revenue increases. Moreover, investment growth causes a cost reduction, and therefore, product prices could fall. However, there is a time gap between the investment and its payback, which allows the costs to rise at the investment moment.

Possible growth of the demand caused by the production quality increases (or pricecutting) leads to more intensive use of the production capacity, gaining greater profit and providing the opportunity to increase investments.

2. Reduction of capital investment reduces the production quality, which leads to a recession in the demand. This change leads to a reduction in the output and, consequently, the gross regional product sinking. Output reduction causes a loss of jobs, and thus, a decrease in available income. Regional and federal authorities can prevent these negative trends by means of investments from their own sources.

3 . Investment collapse, as noted in point 2 , results in a reduction in the output, which causes profits to decrease, and consequently, tax revenues decrease. Decrease of budget revenues enlarges pressure on the government and forces the government to direct additional investment into developing production.

4. Inflation causes a demand decrease that leads to a reduction in the output. It causes higher production costs and further increases in prices.

5. A capital investment increase through a loan leads to a rise in the firms' debt costs (accounts payable growth). Interest on credit payments gives rise to individuals' investment diminution. Thus, if the credit loans and interest on credit are high enough compared with the profit, then the capital investments are reduced. This approach results in reduced production and a fall in employment. In that case, the authorities can undertake sharing the debts to support small, medium and large-scale businesses.

6. Taxes and other dues and fees increase the causes of the rise in production costs. This approach leads to a reduction in the output, which causes a fall in employment. The authorities are obliged to revise the tax rates, tariffs and other duties and fees for the purpose of avoiding unemployment growth.

Sector Population reflects natural and mechanical population movements. The average population, economically active population, and natural and migration increase (decrease).

The direct relation between the gross regional product and the average wage was recognised according to statistical data. An output increase stimulates a wage rise and, respectively, the population income (ceteris paribus). An increase in income leads to an extension in the demand. If inflation will pass ahead of the income increase, then the demand will become reduced. Subsequently the output, profit and investment will decline.

The resulting indicators (level of employment/unemployment, average wage, real wage, per capita monetary income of the population, real population income, per capita gross regional product and demand) allow us to evaluate the standards of living conditionally on the socio-economic policy.

Sector Prices and Taxes contain the main control parameters of the model: tax rates, inflation forecasts, possible options for business taxation (e.g., a simplified tax system for small-scale businesses).

A high inflation level leads to a slump in the real population income. Accordingly, the business profit and investment opportunities decline. Thus, regional budget revenues are 
reduced. A deficit emerges in the case of the old level retention of budget expenditures. State subsidies might cover the deficit, but simultaneously, the level of budget financing increases. This action has a negative influence on the efficiency of the regional management.

The sources of budget revenues and expenditures are reflected in the sector Consolidated Regional Budget. A regional budget is a regional authority's tool for active intervention in business and social spheres and socio-economic development as a whole.

Interrelations, balance equations, and variables' distributions defined at a previous model level are automatically generated on the third level.

This simulation model is the basis of DSS for the management of regional socioeconomic development.

\subsection{The DSS}

DSS is composed of the three essential blocs: the database, simulation model and interface between the user and the remainder of the system.

According to [22], the interface ought to offer the following characteristics: management of different dialog styles, change in the dialog style conditionally on the user's choice, providing data in different forms and types, and supplying flexible decision support.

The interface designed by using iThink permits changing the values of the control parameters, modelling the process retrace in real time, interfering in the modelling process after receiving the appropriate signals, and visualising the consequences of several scenarios on a common graphical field.

The engineered DSS allows us to accomplish economic multivariate scenario stresstesting that depends on crisis factors.

Special attention is devoted to the following problem: how does business influence territory development? It is shown that large-scale business crucially determines the regional economic growth in modern conditions [29].

The time history of the gross regional product depends on the investment index. The more the capital investment in middle-scale and large-scale businesses declines, the more the gross regional product decreases. The dependence between the capital investment and the gross regional product is expressed indirectly, i.e., through a series of equations. Thus, the investment activity impacts on the production output and, hence, on the business revenue, and the aggregate output of the economic sectors determines the gross regional product.

\section{Discussion and conclusions}

Decision-making processes in regional systems are complex and are frequently partitioned into sub-problems [40]. There are various approaches that are developed for the appreciation of regional economic systems' functioning. In spite of the variety, there are several generally accepted approaches that have become popular recently, viz. the economic base, input-output, econometric analysis, simulation, linear and nonlinear programming, and qualitative statistical approaches.

The development of information technologies facilitated the elaboration of an approach based on computer simulation realisation by means of man-made dialogue. Simulation modelling is the basis of multivariate forecasting and complex systems' analysis. This method allows investigating complex dynamic systems (for example, regions, economic sectors). This method's application involves two steps: a) construction of a dynamic simulation model that is complex; and b) fulfilment of an analytical and forecasting 
computation. Simulation modelling permits us to analyse many alternatives and research a stochastic system under uncertainty and perform other experiments.

Models of systems dynamics are used in common differential equations that are balanced, and there are principles and methods of logistics based on optimisation, management, and integrated flows in complex systems.

Computer modelling adaptation in combination with decision making methods, intelligent technologies, and expert methods is auspicious.

In the process of modelling complex systems such as regions, the approach should account for the necessity of 'narrowing' the comprehension of the simulated model due to identifying the most significant socio-economic factors. Because of this fact, regional management becomes aimed at specific goals and objectives (e.g., stimulation of investment activity, rise in budget revenues and so on).

The model presented in this paper is constructed on the principles of systems dynamics and is extensible and adaptive. The above-described toolkit can be adapted for regions of Russian Federation.

Undoubtedly, spheres of regional activity are not limited to the six spheres presented below. The model can be considered to be a basic variant, and it could be further supplemented with blocks, for example, Foreign-economic activity, Ecological situation, and Land use. Adding blocks can help to improve the decision support system and can help in the choice of the most appropriate strategies. The simulation model and DSS based on it permit us to answer the question what if? (e.g., if the investment increases/drops to a certain level, what is the gross regional product/average wage/budget revenue?). This tool allows us to make more reasonable and valid decisions on the basis of the oscillations and analysis of multifarious scenarios of further regional development.

The decision support system for the management of a regional economy is aimed at offering the users (regional authorities, departments, agencies) a flexible and user-friendly tool to assist them in keeping and structuring the information, accomplishing the statistical analysis, and providing a decision aid by obtaining possible scenarios of further regional development conditional on changes in the control parameters. Decision makers can now test their own insights.

Technically, the system is accessible with a standard personal computer. Future research might investigate how this DSS can be used by several decision makers simultaneously.

The article was supported with grant of the President of the Russian Federation, No. MK-218.2017.6.

\section{References}

1. J. Alcamo, Scenarios as tools for international environmental assessments (European Environment Agency, Copenhagen, 2001)

2. S. L. Alter, Decision support systems: current practice and continuing challenges (Addison-Westley Publishing Co., Massachusetts, 1980)

3. P. Antunes, R. Santos, N. Videira, Land Use Policy, 23 (2006)

4. S. A. Aristov, Economics and Mathematical Methods, 43, 3 (2007)

5. P. J. Bacon, J. D. Cain, M. Kozakiewicz et al., Journal of Environmental Assessment Policy and Management, 4, 2 (2002)

6. A. A. Barsegyan, M. S. Kupriyanov, V. V. Stepanenko, I. I. Kholod, Technologies of data analysis: data mining, visual mining, text mining (OLAP, St. Petersburg, 2007)

7. K. A. Feofanov, Vestnik MSTU «Stankin», 4 (2009) 
8. E. Fernandez, J. Navarro, A. Duarte, G. Ibarra, Decision Support Systems, 54, 3 (2013)

9. J. W. Forrester, World dynamics (Wriht-Allen Press, 1971)

10. J. W. Forrester, Industrial dynamics (Pegasus Communications, Waltham, 1961)

11. J. W. Forrester, Urban dynamics (MIT Press, Cambridge, 1969)

12. K. V. Gaidrik, Decision support systems: evolution of concept and some views (http://masters.donntu.edu.ua/2010/fknt/sheptulia/library/article05.htm, 2010)

13. R. Greiner, Mathematics and Computers in Simulation, 64, 1 (2004)

14. B. Hogwood, L. Gunn, Policy analysis for the real world (Oxford,1984)

15. C. W. Holsapple, A. B. Whinston, Decision support systems: a knowledge based approach (West, St. Paul, 1996)

16. G. H. Huang, W. Sun, X. Nie, X. Qin, X. Zhang, Environmental Modelling and Software, 25, 1 (2010)

17. iThink, Systems thinking for business (http://www.iseesystems.com/Softwares /Business/ithinkSoftware.aspx, 2012)

18. P. G. W. Keen, Decision Support Systems, 3 (1987)

19. G. B. Kleiner, Russian economics, 2, 7 (2001)

20. D. A. Kononov, V. V. Kulba, S. S. Kovalevsky, S. A. Kosyachenko, Building scenario spaces and analysis of socio-economic systems' behavior modification (ICS RAS, Moscow, 1999)

21. G. Kou, Y. Shi, S. Wang, Decision Support Systems, 51, 2 (2011)

22. O. I. Larichev, A. B. Petrovsky, Overall Results of Science and Engineering, 21 (1987)

23. D. P. Loucks, Journal of Hydrology, 328 (2006)

24. N. N. Lychkina, III International Conference Proceedings SICPRO'04 (ICS RAS, Moscow, 2005)

25. D. L. Meadows, D. H. Meadows, J. Randers, Beyond the limits: confronting global collapse, envisioning a sustainable future (Chelsea Green Publishing, White River Junction, 1993)

26. Z. Naveh, Landscape Urban Planning, 50 (2000)

27. R. R. Nelson, S. G. Winter, An evolutionary theory of economic change (Delo, Moscow, 2002)

28. H. Nowotny, P. Scott, M. Gibbons, Knowledge creation, diffusion, and use in innovation networks and knowledge clusters: a comparative systems approach across the United States, Europe, and Asia (Praeger Publisher, Westport, 2006)

29. O. Yu. Patrakeeva, Economics and Entrepreneurship, 2 (2012)

30. G. G. Pocheptsov, Theory and Practice of Management, 6 (2004)

31. D. J. Power, Decision support systems: concepts and resources for managers (Quorum Books, Westport, 2002)

32. V. A. Putilov, A. V. Gorokhov, System dynamics of regional development (Murmansk, SRC «Pazori», 2002)

33. M. Quaddus, A. Intrapairot, Decision support systems, 31 (2001)

34. N. Quaranta, A. DeMartini, R. Bellasio, R. Bianconi, M. Marioni, Environmental Modeling and Software, 17 (2002) 
35. J. D. Sterman, Business dynamics: systems thinking and modeling for a complex world (Irwin/McGraw-Hill, 2000)

36. Yu. P. Surmin, System theory and system analysis (MAUP, Tutoria, 2003)

37. B. Tress, G. Tress, Interdisciplinary and transdisciplinary landscape studies: potential and limitations (Wageningen, 2003)

38. B. Tress, G. Tress, Landscape Urban Planning, 64 (2003)

39. I. F. Tsisar, Crises, taxes, inflation, banks (Dialog-MIFI, Moscow, 2009)

40. L. Xue, Y. Zhu, Y. Xue, Mathematical and Computer Modelling, 58 (2013)

41. H. Yuan, A. R. Chini, Y. Lu, L. Shen, Waste Management, 32 (2012) 